\title{
nmT5 - Is parallel data still relevant for pre-training massively multilingual language models?
}

\author{
Mihir Kale* Aditya Siddhant* Rami Al-Rfou \\ Linting Xue Noah Constant Melvin Johnson \\ Google Research
}

\begin{abstract}
Recently, mT5 - a massively multilingual version of T5 - leveraged a unified text-to-text format to attain state-of-the-art results on a wide variety of multilingual NLP tasks. In this paper, we investigate the impact of incorporating parallel data into mT5 pre-training. We find that multi-tasking language modeling with objectives such as machine translation during pretraining is a straightforward way to improve performance on downstream multilingual and cross-lingual tasks. However, the gains start to diminish as the model capacity increases, suggesting that parallel data might not be as essential for larger models. At the same time, even at larger model sizes, we find that pre-training with parallel data still provides benefits in the limited labelled data regime.
\end{abstract}

\section{Introduction}

Recent works have shown that cross-lingual transfer learning in pre-trained multilingual models such as mBERT (Devlin et al., 2019) and XLM-R (Conneau et al., 2020) could be improved further by using parallel data (Conneau and Lample, 2019; $\mathrm{Hu}$ et al., 2020a; Ouyang et al., 2020; Luo et al., 2020). In this paper, we continue this line of work by improving the recent mT5 model (Xue et al., 2020) by leveraging parallel corpora. We experiment with several text-to-text objectives that incorporate parallel data (spanning 198 language pairs) into mT5 pre-training. Our key findings are summarized below:

- In the regime of very small fine-tuning datasets, objectives with parallel data improve results significantly.

- The gain from using parallel data decreases as we scale up the size of the pre-trained model.

${ }^{*}$ Equal Contribution. Please direct correspondence to \{ mihirkale, adisid\} @google.com
- Simple objectives based on neural machine translation (NMT) perform better than the traditionally employed "translation language modeling" (TLM) objective.

\section{Method}

We focus on the mT5-Large model, which is a 24 layer encoder-decoder transformer model and has shown strong performance on a variety of crosslingual benchmarks (Xue et al., 2020). Instead of training a new model from scratch, we start from the publicly available mT5-Large checkpoint - which has been trained for over 1 trillion tokens - and do a second stage pre-training with a mix of monolingual and parallel data.

\subsection{Objectives}

The mT5 - multilingual version of T5 (Raffel et al., 2020) - series of models were pre-trained on a multilingual version of the $\mathrm{C} 4$ corpus with a masked language modeling "span-corruption" objective (Raffel et al., 2020), where the encoder is fed a chunk of text with random spans replaced with a mask token, and the decoder must reconstruct the masked-out tokens. One of their primary distinctions is the use of a unified "text-to-text" format for all text-based NLP problems.

In keeping with the text-to-text format, we experiment with the following objectives to incorporate parallel data into pre-training:

- TLM - A text-to-text version of translation language modeling, proposed by Conneau and Lample (2019) and subsequently used in several prior works for encoder only pre-training. We trivially extend it to the encoder-decoder setting.

- NMT - Standard machine translation. The input is the source text and the target is its 


Denoised NMT-LM

Figure 1: Example source and targets for different text-to-text style pre-training objectives incorporating parallel data. All objectives except TLM specify target language in the source sentence.

translation. A language code is prefixed to the input to inform the model of the target language (Johnson et al., 2017).

- Denoised-NMT - Similar to NMT, but we additionally mask spans in the source sentence. The model must now learn to implicitly perform language modeling of the source language while translating into the target language.

- Denoised-NMT+LM - Similar to DenoisedNMT, but instead of implicit language modeling, the model must explicitly predict the source text in addition to the translation. The target is a concatenation of the translation and source sentence, while the input is the masked source sentence.

We refer to the model trained with the standard NMT objective as nmT5.

\section{Experiment Setup}

Pre-training datasets For pre-training we use monolingual data from mC4 (Xue et al., 2020) and parallel data from OPUS-100 (Zhang et al., 2020). OPUS-100 is a dataset of $55 \mathrm{M}$ translations covering 100 languages (198 language pairs, either into or from English). The mC4 corpus consists of unlabeled web text covering 101 languages, of which 81 overlap with the OPUS-100 languages.

Fine-tuning datasets For downstream evaluation, we use the following four tasks:

- TyDi QA (Clark et al., 2020) - The GoldP subtask, which corresponds to extractive question answering. The input is a passage and a question, with the answer being a span from the passage.

- MTOP (Li et al., 2020) - Multilingual TaskOriented Parsing. The task is one of structured

\begin{tabular}{l|rrc}
\hline Dataset & Langs & Train size & Setting \\
\hline TyDi QA & 9 & $3.7 \mathrm{~K}$ & zero-shot \\
MTOP & 6 & $22 \mathrm{~K}$ & zero-shot \\
WikiAnn NER & 40 & $20 \mathrm{~K}$ & zero-shot \\
WikiLingua & 18 & $660 \mathrm{~K}$ & multilingual \\
\hline
\end{tabular}

Table 1: Statistics of datasets used in the paper.

prediction, where user queries must be parsed into a tree, capturing the domain, intent and slots.

- WikiAnn NER (Pan et al., 2019) - Named entity recognition task covering 40 languages featured in the XTREME benchmark (Hu et al., 2020b). There are 4 categories of entities - location, person, organization and miscellaneous.

- WikiLingua (Ladhak et al., 2020) - A recently introduced cross-lingual summarization dataset, where a document from an arbitrary language must be summarized in English. Since the dataset does not come with training and evaluation splits, we randomly create validation and test sets of 1000 examples each, and the rest of the data is used for training.

Table 1 lists further details of each dataset. Following Xue et al. (2020), all tasks are cast into the text-to-text format. The evaluation for TyDi QA, MTOP and NER is done in the zero-shot setting, where the model is trained on the English data and evaluated on all languages. Since zero-shot cross-lingual language generation is much harder, for WikiLingua we train the model in a multilingual setting, using available training data for all languages.

Hyperparameters Pre-training is done with a batch size of $1 \mathrm{M}$ tokens and fine-tuning with 131,072 tokens, with a constant learning rate of 


\begin{tabular}{l|ccccc}
\hline $\begin{array}{l}\text { Model } \\
\text { (Metric) }\end{array}$ & $\begin{array}{c}\text { TyDi QA } \\
\text { (F1/EM) }\end{array}$ & $\begin{array}{c}\text { MTOP } \\
(\text { EM) }\end{array}$ & $\begin{array}{c}\text { NER } \\
\text { (F1) }\end{array}$ & $\begin{array}{c}\text { WikiLingua } \\
\text { (ROUGE-L) }\end{array}$ & Avg. \\
\hline mT5 & $66.3 / 49.8$ & 43.7 & 58.4 & 25.2 & 46.3 \\
+MLM (additional 100K steps) & $71.3 / 55.6$ & 48.6 & 59.9 & 26.1 & 49.5 \\
+MLM+TLM & $71.1 / 54.6$ & 48.6 & 61.4 & 26.1 & 49.7 \\
+MLM+NMT & $75.1 / 60.1$ & 57.7 & 61.4 & 27.4 & 53.5 \\
+MLM+denoised NMT & $75.3 / 60.2$ & 56.5 & 61.5 & 27.4 & 53.3 \\
+MLM+denoised NMT-LM & $75.0 / 59.4$ & 56.0 & 62.4 & 26.9 & 53.1 \\
\hline
\end{tabular}

Table 2: Results are averaged across all the languages in each dataset. We report F1/EM for QA, exact match accuracy (EM) for structured prediction, ROUGE-L (Lin, 2004) for summarization and F1 for NER. Each score is the median over five runs. The final columns lists the average of all the scores. Refer to Appendix A for scores on individual languages.

0.001. Starting from publicly available mT5-Large checkpoints, we further pre-train for $100 \mathrm{~K}$ steps with a mix of monolingual and parallel objectives. The parallel data is mixed into monolingual data at a $10 \%$ ratio, which amounts to roughly 4 passes over the OPUS-100 corpus. Examples from each language pair are sampled using the same language sampling distribution as Xue et al. (2020), with alpha $=0.3$. For downstream tasks, we fine-tune for $10 \mathrm{~K}$ steps for TyDiQA, MTOP, NER and $25 \mathrm{~K}$ for WikiLingua, since it is a much larger dataset. Checkpoint selection is done based on the validation set.

Baselines Our first baseline is the publicly available mT5-Large model (1.3 billion parameters). For a fair comparison, we also experiment with an mT5 model further pre-trained for 100k steps with only monolingual data from $\mathrm{mC} 4$ (see row 2 : mT5+MLM in Table 2). This lets us assess whether improvements stem from using parallel data or just pre-training for longer.

\section{Results}

We report results in table 2. Overall, adding parallel data through neural machine translation objectives improves scores for all 4 tasks, with the NMT objective performing the best.

Simply pre-training mT5 for longer with just monolingual data (MLM) leads to improved scores for all tasks. The TLM objective is not be able to effectively leverage the parallel data and performs on par with MLM. On the other hand, our three NMTbased objectives show gains over MLM across all tasks. Among these, NMT and Denoised-NMT are the best and perform similarly, while DenoisedNMT+LM fares slightly worse. Averaged across all tasks, NMT and Denoised-NMT outperform
MLM by 4 points.

\subsection{Model size}

Xue et al. (2020) find that cross-lingual performance of language models increases monotonically with model size. To study the impact of model capacity, we also experiment with larger model sizes. Even at the XL size (3.7B params, $3 \times$ larger than mT5-Large), we observe gains for all tasks with nmT5 (Table 3). However, the magnitude of the gains is largely diminished, hinting that the need for parallel data reduces as model capacity increases. This finding is particularly promising for low-resource languages, where it is difficult to obtain high-quality parallel data.

At the same time, nmT5-Large substantially reduces the performance gap between mT5-Large and mT5-XL, covering $70 \%$ of the headroom. Since bigger models are expensive to train and even more expensive to deploy, this opens up avenues for effectively using parallel data to improve performance of smaller language models. Turc et al. (2019) found that pre-training student models before model distillation is helpful, and using parallel data to improve student pre-training is another interesting avenue of future work.

\begin{tabular}{l|ccccc}
\hline Model & TyDi QA & MTOP & NER & WikiLingua & Avg. \\
\hline mT5-Large & $66.3 / 49.8$ & 43.7 & 58.4 & 25.2 & 46.3 \\
nmT5-Large & $75.1 / 60.1$ & 57.7 & 61.4 & 27.4 & 53.5 \\
$\Delta$ & $8.8 / 10.3$ & 14.0 & 3.0 & 2.2 & 7.2 \\
\hline mT5-XL & $77.8 / 61.8$ & 63.4 & 65.5 & 27.9 & 56.7 \\
nmT5-XL & $78.4 / 63.3$ & 64.9 & 66.2 & 28.4 & 57.6 \\
$\Delta$ & $0.6 / 1.5$ & 1.5 & 0.7 & 0.5 & 0.9 \\
\hline
\end{tabular}

Table 3: Impact of model size on nmT5's performance. 


\begin{tabular}{l|lcc}
\hline Model & Few-Shot (100) & Low (3.7K) & High (80K) \\
\hline mT5-Large & $33.1 / 23.6$ & $66.3 / 49.8$ & $78.1 / 64.8$ \\
nmT5-Large & $48.8 / 37.1$ & $75.1 / 60.1$ & $78.2 / 65.5$ \\
$\Delta$ & $15.7 / 13.5$ & $8.8 / 10.3$ & $0.1 / 0.7$ \\
\hline mT5-XL & $45.0 / 31.7$ & $77.8 / 61.8$ & $78.7 / 65.8$ \\
nmT5-XL & $57.2 / 44.4$ & $78.4 / 63.3$ & $79.7 / 67.0$ \\
$\Delta$ & $12.2 / 12.7$ & $0.6 / 1.5$ & $1.0 / 1.2$ \\
\hline
\end{tabular}

Table 4: Performance on the TyDi QA eval set when fine-tuned in the few-shot (100 examples from TyDi QA English), low (full TyDi QA English with 3.7K examples) and high data regime (SQuAD English with 80K examples).

\subsection{Limited labeled data}

The TyDi QA dataset has only 3.7K English training examples. To study the impact of the size of fine-tuning data, we run experiments in two additional settings: a few-shot regime and a high data regime. Few-shot uses just 100 randomly sampled training examples, while for the latter we use the much larger SQuAD corpus (Rajpurkar et al., 2016), which consists of 80k examples.

When fine-tuned with SQuAD, nmT5 performs slightly better than mT5 for both Large and XL model sizes. However, in the few-shot setting, nmT5-Large improves over mT5-Large by 15 points. Even at the XL size, nmT5 is over 10 points higher than mT5. nmT5-Large even outperforms the much larger mT5-XL. Our experiments suggest that pre-training with parallel data is particularly useful in the limited labelled data setting.

\subsection{Mixing ratio}

So far, we have mixed parallel data into monolingual data at a $10 \%$ ratio. To assess how the mixing ratio impacts performance, we compare results with a $50 \%$ mix. With the $50 \%$ mix, average performance is slightly lower, validating our initial choice.

\begin{tabular}{c|ccccc}
\hline Mix & TyDi QA & MTOP & NER & WikiLingua & Avg. \\
\hline $10 \%$ & $75.1 / 60.1$ & 57.7 & 61.4 & 27.4 & 53.5 \\
$50 \%$ & $76.5 / 60.1$ & 53.9 & 62.0 & 26.5 & 52.7 \\
\hline
\end{tabular}

Table 5: Impact of mixing ratio on nmT5.

\subsection{Performance on unseen languages}

We also test downstream performance on languages previously unseen by the models. We randomly pick 30 languages from the WikiAnn NER dataset that are not covered in either $\mathrm{mC} 4{ }^{1}$ or OPUS, and hence none of our models have seen them during pre-training. Table 6 shows nmT5 outperforms mT5 on this subset of languages as well, indicating that the representations of the nmT5 model are better suited for cross-lingual transfer.

\begin{tabular}{l|ccc|c}
\hline Model & ckb & hsb & xmf & "Avg." \\
\hline mT5-Large & 66.5 & 64.8 & 58.4 & 54.9 \\
nmT5-Large & 72.2 & 69.8 & 62.2 & 57.4 \\
$\Delta$ & 5.7 & 5.0 & 3.8 & 2.5 \\
\hline
\end{tabular}

Table 6: Performance on three randomly picked unseen languages. "Avg." is calculated by averaging performance across 30 unseen languages.

\section{Related Work}

Pre-trained multilingual models such as mBERT and XLM-R have shown to be effective at crosslingual transfer learning (Devlin et al., 2019; Conneau et al., 2020). Subsequently, many attempts have leveraged parallel data to improve crosslingual capability of these models. Conneau and Lample (2019) proposed translation language modeling (TLM), to encourage the model to align representations across languages. Alternating language modeling (Yang et al., 2020) and back-translation masked language modeling (Ouyang et al., 2020) used code-switched sentences and back-translation respectively to utilize parallel data. Other works using parallel data in this line of work include FILTER (Fang et al., 2020), AMBER (Hu et al., 2020a) and, MMTE (Siddhant et al., 2020). A key factor that differentiates this paper from these works is that our pre-trained models use a text-to-text architecture, having both an encoder and a decoder, while the aforementioned models only have the encoder. Other pretrained multilingual encoderdecoder models such as mT5 (Xue et al., 2020), mBART (Liu et al., 2020) and MASS (Song et al., 2019) do not make use of parallel data during pretraining.

\section{Conclusion}

In this work we attempted to improve mT5 pretraining by incorporating parallel data. We experimented with various text-to-text objectives and found that multi-tasking with the standard neural machine translation objective during pre-training

\footnotetext{
${ }^{1}$ Subject to precision of language ID models used for $\mathrm{mC} 4$.
} 
leads to improved cross-lingual transfer. The improvements from parallel data are most pronounced in the limited labeled data scenario. Our experiments also indicate that smaller models, with the help of parallel data, can approach the performance of larger ones, while also suggesting that the need for parallel data is lesser as the model capacity increases.

\section{References}

Jonathan H Clark, Eunsol Choi, Michael Collins, Dan Garrette, Tom Kwiatkowski, Vitaly Nikolaev, and Jennimaria Palomaki. 2020. Tydi qa: A benchmark for information-seeking question answering in ty pologically di verse languages. Transactions of the Association for Computational Linguistics, 8:454-470.

Alexis Conneau, Kartikay Khandelwal, Naman Goyal, Vishrav Chaudhary, Guillaume Wenzek, Francisco Guzmán, Édouard Grave, Myle Ott, Luke Zettlemoyer, and Veselin Stoyanov. 2020. Unsupervised cross-lingual representation learning at scale. In Proceedings of the 58th Annual Meeting of the Association for Computational Linguistics, pages 84408451.

Alexis Conneau and Guillaume Lample. 2019. Crosslingual language model pretraining. In Advances in Neural Information Processing Systems.

Jacob Devlin, Ming-Wei Chang, Kenton Lee, and Kristina Toutanova. 2019. BERT: Pre-training of deep bidirectional transformers for language understanding. In Proceedings of the 2019 Conference of the North American Chapter of the Association for Computational Linguistics: Human Language Technologies, Volume 1 (Long and Short Papers), pages 4171-4186, Minneapolis, Minnesota. Association for Computational Linguistics.

Yuwei Fang, Shuohang Wang, Zhe Gan, Siqi Sun, and Jingjing Liu. 2020. Filter: An enhanced fusion method for cross-lingual language understanding. arXiv preprint arXiv:2009.05166.

Junjie Hu, Melvin Johnson, Orhan Firat, Aditya Siddhant, and Graham Neubig. 2020a. Explicit alignment objectives for multilingual bidirectional encoders. arXiv preprint arXiv:2010.07972.

Junjie Hu, Sebastian Ruder, Aditya Siddhant, Graham Neubig, Orhan Firat, and Melvin Johnson. 2020b. Xtreme: A massively multilingual multi-task benchmark for evaluating cross-lingual generalisation. In International Conference on Machine Learning.

M. Johnson, Mike Schuster, Quoc V. Le, M. Krikun, Y. Wu, Z. Chen, Nikhil Thorat, F. Viégas, M. Wattenberg, G. S. Corrado, Macduff Hughes, and J. Dean.
2017. Google's multilingual neural machine translation system: Enabling zero-shot translation. Transactions of the Association for Computational Linguistics, 5:339-351.

Faisal Ladhak, Esin Durmus, Claire Cardie, and K. McKeown. 2020. Wikilingua: A new benchmark dataset for cross-lingual abstractive summarization. ArXiv, abs/2010.03093.

Haoran Li, A. Arora, Shuohui Chen, Anchit Gupta, Sonal Gupta, and Yashar Mehdad. 2020. Mtop: A comprehensive multilingual task-oriented semantic parsing benchmark. ArXiv, abs/2008.09335.

Chin-Yew Lin. 2004. Rouge: A package for automatic evaluation of summaries. In Text summarization branches out, pages 74-81.

Yinhan Liu, Jiatao Gu, Naman Goyal, Xian Li, Sergey Edunov, Marjan Ghazvininejad, Mike Lewis, and Luke Zettlemoyer. 2020. Multilingual denoising pre-training for neural machine translation. Transactions of the Association for Computational Linguistics, 8:726-742.

Fuli Luo, Wei Wang, Jiahao Liu, Yijia Liu, Bin Bi, Songfang Huang, Fei Huang, and Luo Si. 2020. Veco: Variable encoder-decoder pre-training for cross-lingual understanding and generation. arXiv preprint arXiv:2010.16046.

Xuan Ouyang, Shuohuan Wang, Chao Pang, Yu Sun, Hao Tian, Hua Wu, and Haifeng Wang. 2020. Ernie$\mathrm{m}$ : Enhanced multilingual representation by aligning cross-lingual semantics with monolingual corpora. arXiv preprint arXiv:2012.15674.

Xiaoman Pan, Thamme Gowda, Heng Ji, Jonathan May, and Scott Miller. 2019. Cross-lingual joint entity and word embedding to improve entity linking and parallel sentence mining. In Proceedings of the 2nd Workshop on Deep Learning Approaches for Low-Resource NLP (DeepLo 2019), pages 5666, Hong Kong, China. Association for Computational Linguistics.

Colin Raffel, Noam Shazeer, Adam Roberts, Katherine Lee, Sharan Narang, Michael Matena, Yanqi Zhou, Wei Li, and Peter J. Liu. 2020. Exploring the limits of transfer learning with a unified text-totext transformer. Journal of Machine Learning Research, 21(140):1-67.

Pranav Rajpurkar, Jian Zhang, Konstantin Lopyrev, and Percy Liang. 2016. Squad: 100, 000+ questions for machine comprehension of text. In EMNLP.

Aditya Siddhant, Melvin Johnson, Henry Tsai, Naveen Ari, Jason Riesa, Ankur Bapna, Orhan Firat, and Karthik Raman. 2020. Evaluating the cross-lingual effectiveness of massively multilingual neural machine translation. In Proceedings of the AAAI Conference on Artificial Intelligence. 
Kaitao Song, Xu Tan, Tao Qin, Jianfeng Lu, and TieYan Liu. 2019. Mass: Masked sequence to sequence pre-training for language generation. In International Conference on Machine Learning.

Iulia Turc, Ming-Wei Chang, Kenton Lee, and Kristina Toutanova. 2019. Well-read students learn better: On the importance of pre-training compact models. arXiv: Computation and Language.

Linting Xue, Noah Constant, Adam Roberts, Mihir Kale, Rami Al-Rfou, Aditya Siddhant, Aditya Barua, and Colin Raffel. 2020. mt5: A massively multilingual pre-trained text-to-text transformer. arXiv preprint arXiv:2010.11934.

Jian Yang, Shuming Ma, Dongdong Zhang, ShuangZhi Wu, Zhoujun Li, and Ming Zhou. 2020. Alternating language modeling for cross-lingual pre-training. In Proceedings of the AAAI Conference on Artificial Intelligence.

Biao Zhang, Philip Williams, Ivan Titov, and Rico Sennrich. 2020. Improving massively multilingual neural machine translation and zero-shot translation. In Proceedings of the 58th Annual Meeting of the Association for Computational Linguistics. 


\section{A Per-Language Results on All Tasks}

\begin{tabular}{l|ccccc}
\hline & en & ar & bn & fi & id \\
\hline mt5 & $75.0 / 63.0$ & $68.9 / 51.4$ & $54.5 / 37.2$ & $70.4 / 54.6$ & $74.3 / 57.0$ \\
+MLM & $78.5 / 68.2$ & $76.1 / 59.9$ & $59.0 / 40.7$ & $73.5 / 61.0$ & $76.7 / 60.0$ \\
+MLM+TLM & $77.3 / 67.0$ & $75.7 / 57.2$ & $61.7 / 39.8$ & $73.3 / 59.0$ & $77.0 / 60.0$ \\
+MLM+NMT & $78.4 / 69.3$ & $78.9 / 63.1$ & $74.0 / 54.9$ & $77.0 / 64.8$ & $79.9 / 64.8$ \\
+MLM+denoised NMT & $78.7 / 68.6$ & $79.8 / 64.7$ & $72.6 / 53.1$ & $77.2 / 64.2$ & $79.8 / 67.6$ \\
+MLM+denoised NMT-LM & $78.2 / 68.2$ & $78.8 / 62.3$ & $69.1 / 49.6$ & $78.2 / 65.7$ & $79.6 / 64.8$ \\
\hline & ko & ru & sw & te & avg \\
\hline mt5 & $57.4 / 47.5$ & $61.5 / 37.1$ & $69.7 / 52.5$ & $65.5 / 48.0$ & $66.3 / 49.8$ \\
+MLM & $64.4 / 55.4$ & $68.6 / 48.9$ & $74.2 / 57.7$ & $71.1 / 48.6$ & $71.3 / 55.6$ \\
+MLM+TLM & $66.5 / 55.8$ & $67.8 / 48.0$ & $73.9 / 57.1$ & $66.5 / 47.5$ & $71.1 / 54.6$ \\
+MLM+NMT & $64.9 / 56.2$ & $72.1 / 51.8$ & $77.2 / 63.1$ & $73.3 / 53.1$ & $75.1 / 60.1$ \\
+MLM+denoised NMT & $67.9 / 58.7$ & $71.9 / 51.5$ & $75.7 / 59.7$ & $74.3 / 53.5$ & $75.3 / 60.2$ \\
+MLM+denoised NMT-LM & $67.8 / 59.4$ & $72.7 / 51.1$ & $76.0 / 59.9$ & $74.4 / 54.0$ & $75.0 / 59.4$ \\
\hline
\end{tabular}

Table 7: TyDi QA GoldP results (F1/EM) for each language.

\begin{tabular}{l|lllllll}
\hline & en & de & es & fr & hi & th & avg \\
\hline mt5 & 83.5 & 41.2 & 45.4 & 43.3 & 21.3 & 27.5 & 43.7 \\
+MLM & 83.3 & 44.5 & 46.3 & 51.8 & 31.9 & 34.0 & 48.6 \\
+MLM+TLM & 85.0 & 42.4 & 47.5 & 49.6 & 31.8 & 35.2 & 48.6 \\
+MLM+NMT & 86.1 & 55.1 & 59.0 & 61.7 & 42.2 & 42.1 & 57.7 \\
+MLM+denoised NMT & 85.8 & 51.6 & 55.2 & 59.5 & 42.7 & 43.9 & 56.5 \\
+MLM+denoised NMT-LM & 85.9 & 51.9 & 55.0 & 57.0 & 44.1 & 41.9 & 56.0 \\
\hline
\end{tabular}

Table 8: MTOP results (EM) for each language. 


\begin{tabular}{l|llllllllllllll}
\hline & en & af & ar & bg & bn & de & el & es & et & eu & fa & fi & fr & he \\
\hline mt5 & 80.5 & 64.5 & 47.7 & 57.2 & 66.5 & 67.0 & 63.9 & 62.0 & 59.0 & 45.5 & 41.4 & 56.9 & 76.7 & 45.1 \\
+MLM & 81.4 & 65.1 & 50.2 & 55.2 & 69.3 & 68.6 & 66.9 & 70.5 & 62.8 & 46.6 & 44.9 & 58.9 & 76.6 & 46.4 \\
+MLM+TLM & 82.4 & 65.6 & 48.8 & 67.2 & 72.2 & 70.1 & 70.8 & 72.6 & 61.2 & 47.5 & 47.1 & 61.4 & 78.7 & 48.0 \\
+MLM+NMT & 82.2 & 64.2 & 56.7 & 61.0 & 69.1 & 70.5 & 64.6 & 66.3 & 66.2 & 49.3 & 48.9 & 60.6 & 78.4 & 46.2 \\
+MLM+denoised NMT & 82.5 & 65.7 & 50.3 & 63.6 & 69.6 & 70.7 & 68.6 & 73.7 & 64.9 & 48.6 & 44.3 & 63.3 & 77.7 & 45.5 \\
+MLM+denoised NMT-LM & 82.9 & 66.1 & 49.5 & 67.7 & 74.5 & 71.1 & 71.3 & 74.2 & 67.1 & 49.9 & 44.8 & 63.2 & 80.2 & 49.6 \\
\hline & hi & hu & id & it & ja & jv & ka & kk & ko & ml & mr & ms & my & nl \\
\hline mt5 & 66.8 & 57.7 & 44.9 & 75.4 & 36.0 & 46.0 & 53.0 & 22.5 & 29.5 & 44.8 & 38.6 & 65.5 & 27.0 & 77.3 \\
+MLM & 66.5 & 61.4 & 46.2 & 76.4 & 35.8 & 49.0 & 53.6 & 23.7 & 31.4 & 46.0 & 39.3 & 67.4 & 33.0 & 78.5 \\
+MLM+TLM & 69.6 & 61.9 & 47.2 & 76.7 & 37.3 & 51.0 & 59.4 & 29.3 & 30.7 & 48.2 & 42.1 & 70.2 & 29.0 & 80.4 \\
+MLM+NMT & 69.8 & 61.7 & 46.1 & 77.3 & 34.5 & 53.0 & 55.2 & 27.0 & 31.4 & 43.0 & 46.7 & 69.0 & 27.0 & 78.9 \\
+MLM+denoised NMT & 65.8 & 63.0 & 46.6 & 77.6 & 37.0 & 54.0 & 58.3 & 26.4 & 29.8 & 44.8 & 42.1 & 64.3 & 30.0 & 80.2 \\
+MLM+denoised NMT-LM & 67.7 & 64.4 & 48.1 & 77.9 & 39.2 & 49.0 & 59.4 & 30.0 & 31.4 & 47.4 & 36.4 & 71.0 & 34.0 & 80.2 \\
\hline & $\mathrm{pt}$ & $\mathrm{ru}$ & $\mathrm{sw}$ & $\mathrm{ta}$ & $\mathrm{te}$ & $\mathrm{th}$ & $\mathrm{tl}$ & $\mathrm{tr}$ & $\mathrm{ur}$ & $\mathrm{vi}$ & yo & zh & avg & \\
\hline m+5 & 73.1 & 48.4 & 66.8 & 39.9 & 37.9 & 8.5 & 77.8 & 57.6 & 45.1 & 76.4 & 58.0 & 41.8 & 58.4 & \\
+MLM & 75.5 & 47.3 & 64.5 & 40.5 & 38.0 & 9.2 & 76.9 & 56.5 & 51.7 & 76.9 & 59.0 & 41.8 & 59.9 & \\
+MLM+TLM & 76.3 & 58.8 & 66.3 & 40.2 & 41.2 & 8.8 & 76.9 & 62.0 & 43.0 & 79.6 & 56.0 & 43.5 & 61.4 & \\
+MLM+NMT & 75.5 & 56.0 & 65.8 & 40.3 & 41.6 & 8.0 & 78.7 & 60.3 & 57.0 & 79.8 & 63.0 & 41.0 & 61.4 & \\
+MLM+denoised NMT & 75.5 & 58.9 & 66.2 & 40.4 & 40.4 & 7.9 & 78.7 & 60.5 & 50.0 & 80.3 & 64.0 & 41.4 & 61.5 \\
+MLM+denoised NMT-LM & 78.6 & 60.9 & 65.6 & 40.6 & 40.9 & 9.1 & 77.0 & 63.1 & 53.5 & 79.8 & 60.0 & 45.5 & 62.4 \\
\hline
\end{tabular}

Table 9: WikiAnn NER results (F1) for each language.

\begin{tabular}{l|cccccccccc}
\hline & en & ar & cs & de & es & fr & hi & id & it & ja \\
\hline mt5 & 29.2 & 23.2 & 22.4 & 25.0 & 25.3 & 24.6 & 25.2 & 25.3 & 24.1 & 26.2 \\
+MLM & 30.0 & 24.0 & 22.9 & 26.0 & 26.6 & 25.5 & 26.1 & 25.8 & 24.9 & 27.8 \\
+MLM+TLM & 30.0 & 24.4 & 23.1 & 25.6 & 26.3 & 25.6 & 26.4 & 25.8 & 25.1 & 27.6 \\
+MLM+NMT & 31.5 & 25.7 & 24.0 & 27.0 & 27.5 & 26.4 & 27.7 & 27.0 & 25.8 & 29.5 \\
+MLM+denoised NMT & 31.3 & 25.7 & 24.7 & 27.3 & 27.5 & 26.8 & 27.8 & 27.2 & 25.8 & 29.2 \\
+MLM+denoised NMT-LM & 30.8 & 25.0 & 23.7 & 26.5 & 27.1 & 26.3 & 27.3 & 26.7 & 25.6 & 28.7 \\
\hline & ko & nl & pt & ru & th & tr & vi & zh & avg & \\
\hline mt5 & 23.8 & 25.7 & 24.6 & 23.9 & 25.3 & 30.9 & 22.9 & 25.8 & 25.2 & \\
+MLM & 25.2 & 26.5 & 25.3 & 24.6 & 27.1 & 31.1 & 23.2 & 27.1 & 26.1 & \\
+MLM+TLM & 24.7 & 26.6 & 25.2 & 24.4 & 26.5 & 31.3 & 23.3 & 27.0 & 26.1 & \\
+MLM+NMT & 26.7 & 27.7 & 26.3 & 25.9 & 28.6 & 34.1 & 23.9 & 28.1 & 27.4 & \\
+MLM+denoised NMT & 26.6 & 28.0 & 25.9 & 25.8 & 28.3 & 33.4 & 24.3 & 28.4 & 27.4 & \\
+MLM+denoised NMT-LM & 25.9 & 27.4 & 25.6 & 24.9 & 27.3 & 33.1 & 23.8 & 27.8 & 26.9 & \\
\hline
\end{tabular}

Table 10: Wikilingua results (Rouge-L) for each language. 


\begin{tabular}{l|ccccccccccc}
\hline & ace & arz & ast & ba & ce & ckb & csb & eml & fur & gan & gn \\
\hline mt5-Large & 44.8 & 50.8 & 83.3 & 38.1 & 21.7 & 66.5 & 56.7 & 39.8 & 64.2 & 42.1 & 48.2 \\
nmt5-Large & 46.7 & 53.6 & 84.8 & 43.7 & 28.3 & 72.2 & 58.1 & 41.9 & 65.6 & 41.2 & 51.0 \\
\hline & hsb & ia & jbo & lij & lmo & min & nap & nov & pdc & pms & pnb \\
\hline mt5-Large & 64.8 & 63.2 & 42.1 & 46.3 & 69.8 & 39.1 & 62.2 & 62.1 & 48.1 & 81.5 & 61.1 \\
nmt5-Large & 69.8 & 62.4 & 43.6 & 43.0 & 72.0 & 45.5 & 61.7 & 66.7 & 51.2 & 83.5 & 55.4 \\
\hline & rm & sa & tl & qu & vec & vep & vls & xmf & avg & & \\
\hline mt5-Large & 64.1 & 17.4 & 78.6 & 27.5 & 66.9 & 63.6 & 74.4 & 58.4 & 54.9 & & \\
nmt5-Large & 67.6 & 23.0 & 79.4 & 35.6 & 66.7 & 68.0 & 77.5 & 62.2 & 57.4 & & \\
\hline
\end{tabular}

Table 11: WikiAnn NER results on unseen languages. Refer to section 4.4 